\title{
The ALOHA Systems in Shadowed Mobile Radio Channels with Slow or Fast Fading
}

\author{
ASRAR U. H. SHEIKH, SENIOR MEMBER, IEEE, YU-DONG YAO, MEMBER, IEEE, AND \\ XIAOPING WU, STUDENT MEMBER, IEEE
}

\begin{abstract}
The throughput performance of ALOHA systems in mobile radio channels can be improved due to the capture phenomenon, meaning that the strongest packet may capture the receiver even in the presence of other overlapping packets. In this paper, the influence of receiver capture on the performance of ALOHA in the presence of shadowing is investigated. The combined effect of Rayleigh fading, log-normal shadowing, and spatial distribution of mobile users is also studied. The capture phenomenon results in substantial improvement in system throughput under slow fading. Under fast fading conditions, the throughput can also be increased especially when error-correction coding is used.
\end{abstract}

\section{INTRODUCTION}

$\mathrm{D}$ URING THE EARLY 1970's, the ALOHA protocol was a topic of considerable research because of its attractiveness in providing reasonable efficiency in random access packet radio networks [1], [2]. It is well known that the maximum realizable throughput is $1 / 2 e$ and $1 / e$ for pure and slotted ALOHA, respectively. These results are obtained by assuming that the channel is noiseless and all packets arrive at the receiver with the same power level. When two or more packets arrive at the receiver during overlapping time, they collide and all packets involved are destroyed. In a practical packet radio network, the transmitted packets experience not only noise but also fading, with the result that the receiver may fail to detect the faded packet even though there is no collision [3]. The fact that independently faded packets, with different signal strength, arrive at the receiver makes the capture effect possible (the strongest signal may capture the receiver), so that the probability of mutual destruction of packets is reduced [4]. This results in an increase in the system throughput [5]-[7].

In the mobile radio environment, the power level difference between packets may arise due to Rayleigh fading (multipath propagation), shadowing (by buildings and hills) and the near/far effect (the spatial distribution of mobile users). The ALOHA network is expected to benefit from all three factors. The performance improvement of ALOHA

Manuscript received June 24, 1989; revised September 11, 1989 and March 26, 1990.

A. U. H. Sheikh is with the Department of Systems and Computer Engineering, Carleton University, Ottawa, ON, Canada K1S 5B6.

Y. D. Yao was with Southeast University, Nanjing, Jiangsu, China. He is now with the Department of Systems and Computer Engineering, Carleton University, Ottawa, ON, Canada K1S 5B6.

$X$. Wu was with Concordia University, Montreal, PQ, Canada. She is now with the Department of Systems and Computer Engineering, Carleton University, Ottawa, ON, Canada K1S 5B6.

IEEE Log Number 9038882 systems in Rayleigh fading channels is discussed in [6] and [7], the near/far effect on ALOHA systems is studied in [8] and [9], and the combined effect of fading and the near/far phenomenon has also been studied in [5], [6]. However, the effect of shadowing on ALOHA systems has not been well studied and reported except in two recent publications by Prasad and Arnbak [10], [11] in which fading and shadowing are considered. The present paper investigates the shadowing effect on ALOHA networks. The originality of this work lies in combining the effect of fading, shadowing, and spatial distributions. Furthermore, the fading speed, slow or fast, is brought under consideration. We shall describe a relatively realistic mobile radio ALOHA system model where fading (slow or fast), shadowing and user spatial distributions are included.

In comparing power levels of different packets, it is usually assumed that the power level of a signal remains constant over one packet duration, i.e., the power of each bit in a packet is the same. This assumption has been used earlier [5]- [9], and is considered to be accurate if the users are stationary or are moving very slowly. Also, in some mobile radio systems, the speed of a vehicle could be as high as several tens of kilometers/hour, with the consequence that the channel fades rapidly. In such an environment, the signal power level does not remain constant over the duration of a packet. A receiver operating in a mobile traveling at a moderate speed will experience signal level changes within a packet. A study on the impact of increasing the vehicular speed on the system performance requires second-order statistics of the signal fades. Unfortunately, the second-order statistics of a Rayleigh distributed signal envelope are not available, and it is very difficult, if not impossible, to study the effect of the vehicle speed on ALOHA performance. However, this situation can be resolved, as is the case in this paper, by considering two extreme cases: 1) when the signal remains relatively steady over the duration of the packet, and 2) when two adjacent bits are considered to be faded independently [12]. The latter situation could result when the bits in a packet are interleaved.

ALOHA systems in mobile radio channels have been studied extensively. This paper differs from others in two aspects. First, we consider the combined effect of fading, shadowing, and spatial distributions on ALOHA. Second, the effect of fast fading on ALOHA systems is studied. Section II gives a description of the ALOHA system concerned. Section III analyzes ALOHA systems in the shadowing-only environment without any consideration given to fading and spatial 
distributions of the users. The combined effect of fading, shadowing, and the spatial distribution (the near/far problem) is studied in Section IV and the fast fading effect is studied in Section V. Conclusions are drawn in Section VI.

\section{SYSTEM DESCRIPTION}

\section{A. System Model}

We consider a mobile radio ALOHA system in which packets are transmitted from one vehicle to another via a base station. Only the uplink communication (from mobile users to the central station) is studied in this paper. An infinite number of users generating Poisson distributed data traffic is assumed.

Slotted ALOHA, by allowing transmission only within well-defined time slots, doubles the maximum throughput of pure ALOHA. This paper considers the slotted ALOHA; a similar approach can be used to analyze pure ALOHA schemes.

The mobile channel is characterized by noise, Rayleigh fading, and log-normal shadowing. Because of the spatial distribution of the vehicles, the existence of the near/far effect is also a possibility. A detailed description of these characteristics is provided in the latter sections.

\section{B. Capture Effect}

A desired packet can be received successfully, that is, it captures the receiver in the presence of other overlapping or interfering packets, if its power is larger than the interfering power by a certain margin known as the capture ratio of the receiver. This is the capture effect. The derivation of an expression for the capture probability, the probability that the packet concerned captures the receiver, is one of the major objectives of this paper.

If the desired packet overlaps in time with another packet, the capture probability $P_{\text {capt }}(1)$ is given by

$$
P_{\text {capt }}(1)=\operatorname{Pr}\left(\frac{w_{c}}{w_{u 1}}>R\right) \text {, }
$$

where $w_{c}$ is the power of the packet concerned, $w_{u 1}$ is the power of the interfering packet, and $R$ is the capture ratio. A receiver with $R=1$ is said to have perfect capture, since it can recover the desired packet even if its power is equal to the power of the interfering packet. A receiver with $R=\infty$ cannot capture the desired packet even if the interference power approaches zero.

In the presence of $I(I \geq 1)$ interfering packets (each with power $\left.w_{u i}, i=1,2, \cdots, I\right)$, the capture probability is obtained by comparing $w_{c}$ with total interference power [5], [6], [8],

$$
P_{\text {capt }}^{(1)}(I)=\operatorname{Pr}\left(\frac{w_{c}}{\sum_{i=1}^{I} w_{u i}}>R\right)
$$

Also, if it is assumed that the strongest packet will capture the receiver when the ratio of its power to the power of the next to the strongest packet is above $R$ [2], [7], [9], [13]-[15], the capture probability is

$$
P_{\text {capt }}^{(2)}(I)=\operatorname{Pr}\left(\frac{w_{c}}{w_{u i}}>R, \quad \text { for all } i, 0<i \leq I\right) .
$$

When all the interfering packets are independent, $(2 b)$ can be written as

$$
P_{\text {capt }}^{(2)}(I)=\prod_{i=1}^{I} \operatorname{Pr}\left(\frac{w_{c}}{w_{u i}}>R\right) .
$$

If the distributions of the interfering packets are also identical, we simply have

$$
P_{\text {capt }}^{(2)}(I)=\left[\operatorname{Pr}\left(\frac{w_{c}}{w_{u 1}}>R\right)\right]^{I} .
$$

Under the condition that all the interfering packets are independent and identically distributed, (2b) can be evaluated using (4). However, in general, it has been shown [7] that

$$
\begin{aligned}
& {\left[\operatorname{Pr}\left(\frac{w_{c}}{w_{u 1}}>R\right)\right]^{I} \leq \operatorname{Pr}\left(\frac{w_{c}}{w_{u i}}>R,\right.} \\
& \text { for all } i, 0<i \leq I) .
\end{aligned}
$$

Also, we readily have

$$
\begin{array}{r}
\operatorname{Pr}\left(\frac{w_{c}}{\sum_{i=1}^{I} w_{u i}}>R\right) \leq \operatorname{Pr}\left(\frac{w_{c}}{w_{u i}}>R,\right. \\
\quad \text { for all } i, 0<i \leq I) .
\end{array}
$$

Thus, in general the lower bound of the actual capture probability is provided by (2a) while (2b) gives the upper bound. It is obvious that a receiver which compares the desired packet power with the worst interferer has better capture performance than the one which compares with the total interference power. In this paper, we use (4) to evaluate the capture probability. Note that (4) is a lower bound of (2b) (see (5)) while (2b) is the upper bound of the actual capture probability.

In Appendix I it is shown that (2a) and (4) provide the same results under Rayleigh fading conditions. This indicates that in the Rayleigh fading-only environment, we can simply use (4) to evaluate the lower bound of the capture probability $((2 a))$.

The above equations are derived under the assumption that the signal remains constant over the duration of a packet. This is a valid assumption when the signal fade rate is very slow. If the fade rate becomes high, the signal power may vary over the duration of a packet and all the packets received during a slot should be compared on a bit-by-bit basis. This aspect is studied in Section V.

\section{Throughput}

The channel throughput $S$ is defined as the average number of packets successfully transmitted in a packet length 
duration. It is assumed that each user produces traffic which is Poisson distributed. The total traffic arriving at a receiver from several sources around it will thus have a similar distribution,

$$
p(i)=G^{i} \exp (-G) / i !, \quad i=0,1,2, \cdots
$$

with mean channel traffic $G . P$ is the probability that a packet is successfully transmitted. The channel throughput is

$$
S=G P \text {. }
$$

A packet is transmitted successfully if and only if the following conditions are satisfied. 1) No error occurs due to channel impairments. We may assume that a packet is detected correctly if its power is above some threshold $\left(W_{t}\right)$ and the corresponding probability is denoted as

$$
P_{t}=\operatorname{Pr}\left(w_{c}>W_{t}\right) \text {. }
$$

2) Acknowledgment is received correctly (its probability is $P_{a}$ ). Note that acknowledgment failure results in packet retransmissions. 3) The desired packet captures the receiver. Therefore, we have

$$
\begin{aligned}
P= & p(0) P_{t} P_{a}+p(1) P_{t} P_{a} P_{\text {capt }}(1) \\
& +p(2) P_{t} P_{a} P_{\text {capt }}(2)+\cdots \\
= & P_{t} P_{a} \exp (-G) \sum_{i=0}^{\infty}\left[G P_{\text {capt }}(1)\right]^{i} / i ! \\
= & P_{t} P_{a} \exp (-G) \exp \left[G P_{\text {capt }}(1)\right] .
\end{aligned}
$$

When both $P_{t}$ and $P_{a}$ equal one and $R$ approaches infinity $\left(P_{\text {capt }}(1)\right.$ approaches zero), $(8)$ and (10) reduce to the case of the standard slotted ALOHA in noiseless channels in the absence of the receiver capture effect.

\section{ALOHA Systems in Shadowing Environment}

\section{A. Log-Normal Distribution}

The buildings and hills obstructing the radio signals make them fade such that the signal power $w_{c}$ is log-normally distributed with a probability density function (pdf) given by [16]

$$
p_{w_{c}}\left(w_{c}\right)=\frac{C}{w_{c}} \exp \left[-\frac{\left(\log w_{c}+A\right)^{2}}{B}\right], \quad w_{c}>0
$$

where

$$
\begin{gathered}
C=\frac{\log e}{\sqrt{2 \pi} \sigma}, \\
A=-\log W_{0}+\frac{\sigma^{2}}{2 \log e}, \\
B=2 \sigma^{2},
\end{gathered}
$$

and $W_{0}$ is the mean value of $w_{c}$ and $\sigma$ is the standard deviation in bels.

\section{B. Packet Failure Due to Channel Impairment}

In the absence of interfering packets, a packet may not be detected correctly because of channel impairments such as noise and shadowing. The probability that a packet is transmitted successfully is written as

$$
\begin{aligned}
P_{t} & =\operatorname{Pr}\left(w_{c}>W_{t}\right) \\
& =\int_{W_{t}}^{\infty} p_{w_{c}}\left(w_{c}\right) d w_{c} .
\end{aligned}
$$

After a simple change of the variable $w_{c}$ and assuming $W_{t}=R_{1} W_{0}\left(R_{1} \geq 0\right)$, we have

$$
P_{t}=\frac{1}{\sqrt{\pi}} \int_{L_{1}}^{\infty} \exp \left(-u^{2}\right) d u
$$

where

$$
L_{1}=\frac{\log R_{1}+\frac{\sigma^{2}}{2 \log e}}{\sqrt{2} \sigma} .
$$

Note that (16) approaches one as $R_{1}$ approaches zero. The special case with $R_{1}$ approaching zero means that the packet collision instead of the channel impairments such as noise and shadowing is the major source resulting in packet failure.

\section{Capture Probability}

We can rewrite (1) by considering that $w_{w 1}$ and $w_{c}$ have the same distribution as given in (11). It is further assumed that $w_{u 1}$ and $w_{c}$ have the same mean and standard deviation.

$$
\begin{array}{r}
P_{\text {capt }}(1)=\int_{0}^{\infty}\left[\int_{R x}^{\infty} p_{w_{c}}\left(w_{c}\right) d w_{c}\right] \frac{C}{x} \\
\cdot \exp \left[-\frac{(\log x+A)^{2}}{B}\right] d x .
\end{array}
$$

A simple change of variables results in an expression for $P_{\text {capt }}(1)$ as

$$
\begin{array}{r}
P_{\text {capt }}(1)=\frac{1}{2 \pi \sigma^{2}} \int_{-\infty}^{\infty}\left\{\int_{\log R+x^{\prime}}^{\infty} \exp \left[-\frac{\left(w_{c}^{\prime}+A\right)^{2}}{B}\right] d w_{c}^{\prime}\right\} \\
\cdot \exp \left[-\frac{\left(x^{\prime}+A\right)^{2}}{B}\right] d x^{\prime}
\end{array}
$$

It can be shown that (19) can be reduced to a single integral [17] giving

$$
P_{\text {capt }}(1)=\frac{1}{\sqrt{2 \pi}} \int_{L_{2}}^{\infty} \exp \left(-\frac{u^{2}}{2}\right) d u,
$$

where

$$
L_{2}=\frac{\log R}{\sqrt{2 \sigma}} .
$$

Note that when $R=1,(20)$ is equal to 0.5 .

Inserting (20) into (4), results in the capture probability needed to evaluate channel throughput. 


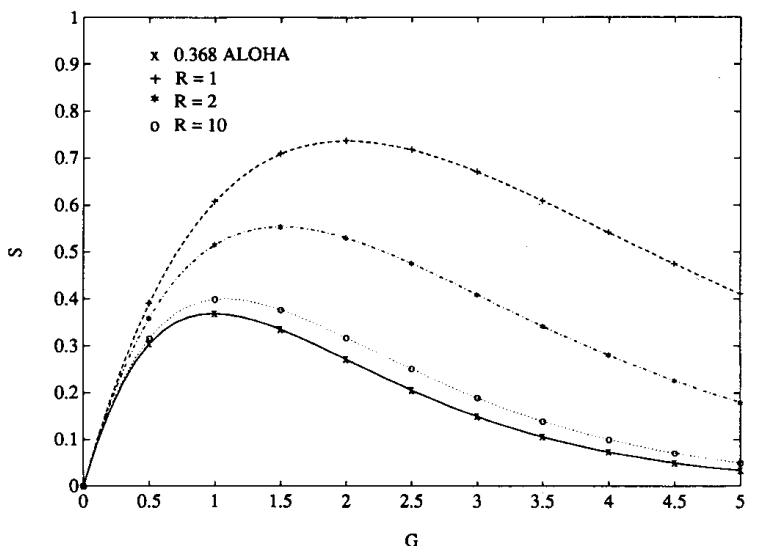

Fig. 1. Throughput curves, assuming shadowing-only environment, $\sigma=5$ $\mathrm{dB}, R_{1}=0$, and $P_{a}=1$

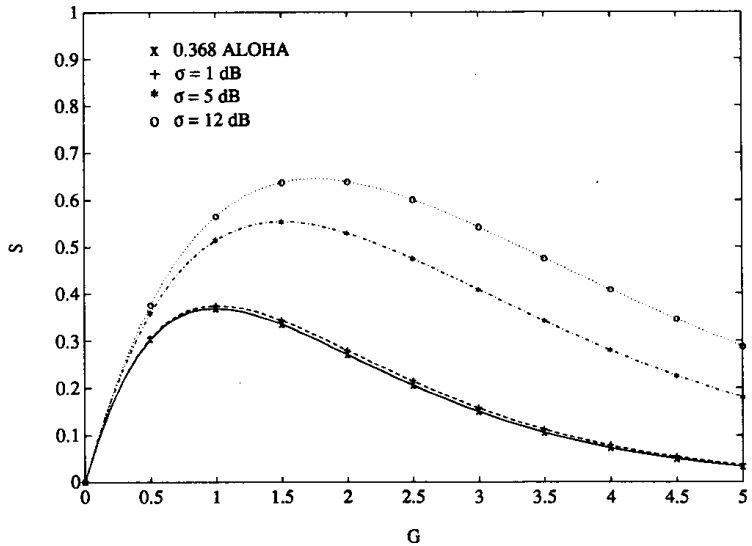

Fig. 2. Throughput curves, assuming shadowing-only environment, $R=2$, $R_{1}=0$, and $P_{a}=1$.

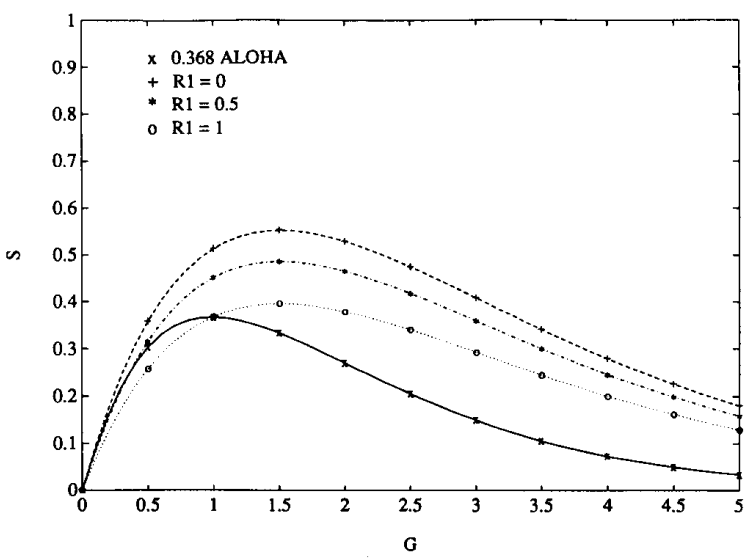

Fig. 3. Throughput curves, assuming shadowing-only environment, $\sigma=5$ $\mathrm{dB}, \boldsymbol{R}=2$, and $\boldsymbol{P}_{a}=1$.

\section{Numerical Results and Discussions}

Numerical computations have been made, resulting throughput curves as shown in Figs. 1-3, and several inferences are drawn from these results. The channel throughput increases with an improvement in receiver capture, or in other words, when $R$ decreases. This is shown in Fig. 1. It is shown in Fig. 2 that significant improvements in throughput are obtained in the presence of shadowing, especially when $\sigma$ is large. As expected, channel impairments (noise and shadowing) reduce the throughput. However, when compared with ALOHA in the absence of channel noise and receiver capture, it is observed that there is still some throughput increase, largely under high-traffic conditions. This is shown in Fig. 3. This means that ALOHA stability is improved in shadowed environment.

\section{Combined Effects of Fading, Shadowing, and Spatial Distribution on ALOHA Systems}

\section{A. Superimposed Rayleigh Fading and Log-Normal Shadowing}

Equation (11) describes the pdf of $w_{c}$, which is log-normally distributed. In this section, we consider the case where the signal envelope is Rayleigh distributed due to fading; that is, $w_{c}$ is exponentially distributed while its mean $W_{c}$ (local mean) is log-normally distributed with an area mean $W_{0}$ and standard deviation $\sigma$. The conditional pdf of $w_{c}$ is then

$$
p_{w_{c} \mid W_{c}}\left(w_{c} \mid W_{c}\right)=\frac{1}{W_{c}} \exp \left(-\frac{w_{c}}{W_{c}}\right), \quad w_{c}>0
$$

and the pdf of $W_{c}$ is

$$
p_{W_{c}}\left(W_{c}\right)=\frac{C}{W_{c}} \exp \left[-\frac{\left(\log W_{c}+A\right)^{2}}{B}\right]
$$

for $W_{c}>0$. Combining (22) and (23), we have, for $w_{c}>0$,

$$
p_{w_{c}}\left(w_{c}\right)=\int_{0}^{\infty} p_{w_{c} \mid w_{c}}\left(w_{c} \mid W_{c}\right) p_{W_{c}}\left(W_{c}\right) d W_{c},
$$

which can be written as

$$
p_{w_{c}}\left(w_{c}\right)=C \int_{0}^{\infty} \exp \left(-w_{c} u\right) \exp \left[-\frac{(A-\log u)^{2}}{B}\right] d u \text {. }
$$

\section{B. Spatial Distribution}

Assume that the base station (or the receiver) is located at the center of a plane and that there is a reference line as shown in Fig. 4. The location of a mobile relative to the base station is determined by the distance to the base station and the angle relative to the reference line. The position of the mobile user is distributed randomly in the plane. The angle $\theta$ can be assumed to be a random variable with a uniform distribution ranging from 0 to $2 \pi$. However, in a typical mobile communication system, the base station uses an omnidirectional antenna, which makes the angle $\theta$ irrelevant to the determination of signal power received at the base station. Thus the signal power is solely determined by the distance $x$. This means that all of the users in a plane may be placed on a single line by rotation of their location through a random angle. This simplifies the problem considerably. Thus we require only the distribution over distance rather than over a plane. Our choice of the probability density for the user distribution is restricted by the near/far phenomenon. We 


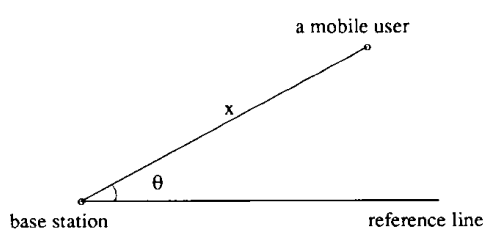

Fig. 4. Location of a mobile user in a plane.

should not allow colocation of the receiver and a transmitter, otherwise the former will be swamped by the latter. Furthermore, a significant number of users should not be allowed at very large distances from the receiver because it imposes limitations on the frequency reuse and connectivity parameters of the network. Many pdf's meet the above requirements. However, according to [18], it is reasonable to assume that the distance between a mobile user and the base station has unimodal distribution. It is also known that for a unimodal pdf the exact functional form of the pdf is not very important [18]. Therefore, following Arnbak et al. [5], [6], we assume that

$$
p_{x}(x)=\frac{5 x_{0}^{4}}{x^{5}} \exp \left[-\frac{5}{4}\left(\frac{x_{0}}{x}\right)^{4}\right], \quad x>0
$$

with a single peak at $x=x_{0}$.

The distance $x$ from any user to the base station is a random variable with a distribution identical to that in (26). Let the ratio of distances between the base station and two users be $r$. As shown in Appendix II, the pdf of $r$ is

$$
p_{r}(r)=\frac{4 r^{3}}{\left(1+r^{4}\right)^{2}}
$$

for $r>0$.

\section{Capture Probability}

When the spatial distribution of the users is considered in the evaluation of the capture probability, the distance of each mobile from the base station must be taken into account by incorporating the distribution given in (27) into the expressions for the probability of capture $((2 a)-(4))$. In other words, we should study $\operatorname{Pr}\left[\left(w_{c} / w_{u 1}\right)\left(r_{u 1} / r_{c}\right)^{\alpha}>R\right]$ instead of $\operatorname{Pr}\left(w_{c} / w_{u 1}>R\right)$. This is obvious for the received signal power at the base station from each user is affected by the propagation losses. The parameter $\alpha$ is determined by the channel propagation model and its value is usually taken to be between two and five. In this paper we consider $\alpha=4$, which characterizes the mobile radio channel. Consider that the desired packet and the interfering packet are transmitted by the users at distances from the base station equal to $r_{c}$ and $r_{u 1}$, respectively, where $r=r_{c} / r_{u 1}$. The interfering packet arrives at the receiver with power $w_{u 1}$ which has the same distribution as $w_{c}$ with local mean $W_{u 1}$ and area mean $W_{0}$. The probability that the considered packet captures the receiver over the interfering packet is

$$
\begin{array}{r}
\operatorname{Pr}\left[\frac{w_{c}}{w_{u 1}}\left(\frac{r_{u 1}}{r_{c}}\right)^{4}>R\right] \\
=\operatorname{Pr}\left(w_{c}>R w_{u 1} r^{4}\right)
\end{array}
$$

$$
\begin{aligned}
& =\int_{0}^{\infty}\left\{C \int_{0}^{\infty}\right. \\
& \left.\cdot \exp (-x u) \exp \left[-\frac{(A-\log u)^{2}}{B}\right] d u\right\} d x \\
& \int_{0}^{\infty} \frac{4 y^{3}}{\left(1+y^{4}\right)^{2}} d y \\
& \int_{R x y^{4}}^{\infty}\left\{C \int_{0}^{\infty}\right. \\
& \left.\cdot \exp \left(-w_{c} v\right) \exp \left[-\frac{(A-\log v)^{2}}{B}\right] d v\right\} d w_{c} \\
& =\int_{0}^{\infty}\left\{C \int_{0}^{\infty}\right. \\
& \left.\cdot \exp (-x u) \exp \left[-\frac{(A-\log u)^{2}}{B}\right] d u\right\} d x \\
& \int_{0}^{\infty} \frac{4 y^{3}}{\left(1+y^{4}\right)^{2}} d y \\
& \cdot C \int_{0}^{\infty} \frac{1}{v} \exp \left(-R x y^{4} v\right) \\
& \cdot \exp \left[-\frac{(A-\log v)^{2}}{B}\right] d v \\
& =4 C^{2} \int_{0}^{\infty} \int_{0}^{\infty} \int_{0}^{\infty} \frac{1}{\left(R y^{4} v+u\right) v} \frac{y^{3}}{\left(1+y^{4}\right)^{2}} \\
& \exp \left[-\frac{(A-\log u)^{2}}{B}\right] \\
& \cdot \exp \left[-\frac{(A-\log v)^{2}}{B}\right] d y d u d v \text {. }
\end{aligned}
$$

Appendix III shows that (28) is reduced to

$$
\begin{aligned}
& \operatorname{Pr}\left[\frac{w_{c}}{w_{u 1}}\left(\frac{r_{u 1}}{r_{c}}\right)^{4}>R\right] \\
&=\frac{1}{\sqrt{\pi}} \int_{-\infty}^{\infty} \frac{\exp \left(-w^{2}\right)}{1-10^{\log R-2 \sigma w}} d w-\frac{C^{2} \sqrt{\pi} B R \ln ^{3} 10}{4 \sqrt{e}} \\
& \cdot \int_{-\infty}^{\infty} \frac{10^{y}}{\left(10^{y}-R\right)^{2}}(y-\log R) \\
& \cdot \exp \left(-\frac{0.5 y^{2}}{B}\right) d y
\end{aligned}
$$




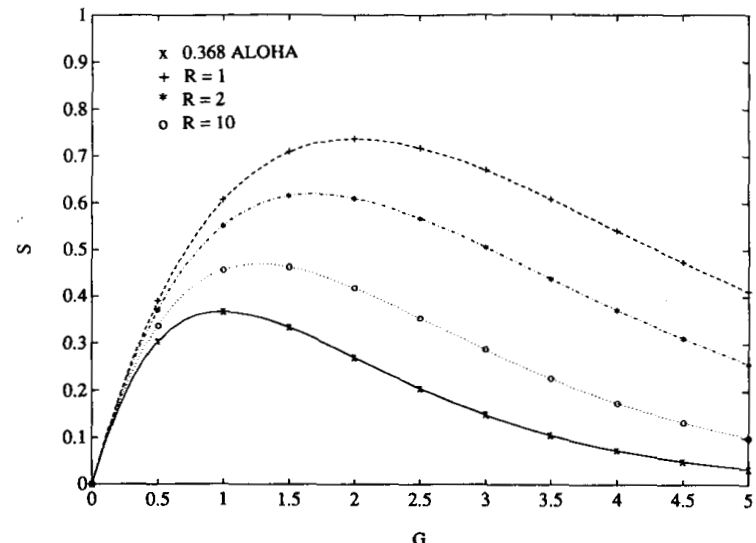

Fig. 5. Throughput curves, considering the combined effect of fading shadowing, and spatial distribution, $\sigma=5 \mathrm{~dB}, R_{1}=0$, and $P_{a}=1$.

In the investigation of the combined effects of spatial distribution and superimposed Rayleigh fading and log-normal shadowing, we have considered that the received composite signal is Rayleigh faded about a log-normally distributed mean and the distance of the mobile from the base station results in an attenuation of the composite signal. This approach is used in the derivation of (28). Note that the effect of spatial distribution on the capture probability can also be studied by incorporating with the area mean for each mobile user. In this approach, the local mean of a signal depends on the distance between the mobile and the base, which reflects the actual physical phenomenon. The two approaches are shown to be equivalent in Appendix IV, while the approach used in this paper provides a simpler derivation.

\section{Numerical Results and Discussions}

It is difficult to solve (29) analytically. Therefore a numerical solution is sought. The results are shown in Fig. 5 . Comparison of Figs. 5 and 1 shows that the composite of Rayleigh fading, log-normal shadowing, and spatial distribution effectively improves the capture effect seen in the shadowing-only environment. This improvement in the capture effect results in higher throughput, a confirmation of the result in [9].

\section{The Fast Fading Effect on AlOHA Systems}

\section{A. Fast Fading Phenomenon}

In the analyses above, a constant signal level is assumed over the duration of a packet; a case where the signal level varies very slowly. In some mobile radio systems, as a result of high vehicular speed, the signal level may change rapidly and the assumption of a constant signal level over a packet length no longer remains valid. A study on the performance variation due to increasing vehicular speed requires secondorder statistics of the signal fades. Unfortunately, for the Rayleigh distributed envelope, second-order statistics are not available. However, we may consider two cases: first, the signal remains constant over the length of a packet, and second, the signal varies continuously such that two adjacent bits of a packet are affected independently. The former case is widely considered in the literature while the latter has not been given enough consideration but is important when interleaving of bits is used in a packet. In previous sections we studied very slowly fading channels in which all of the bits in a packet fade together, a scenario where errors are characterized by bursts. If interleaving is used, the burst error situation can be changed to one where bits in a packet fade independently. We will, however, still assume that the mean of the signal is log-normally distributed but remains constant in one packet duration. For simplicity, the effect of spatial distribution is not taken into consideration in this section.

\section{B. Capture Probability}

Consider $\operatorname{Pr}\left[\left(w_{c} / w_{u 1}\right)>R\right]$ in one-bit duration, in which $w_{c}$ and $w_{u 1}$ are exponentially distributed. We have

$$
\begin{aligned}
\operatorname{Pr}\left(w_{c}>\right. & \left.R w_{u 1}\right) \\
= & \int_{0}^{\infty}\left[\int_{R x}^{\infty} \frac{1}{W_{c}} \exp \left(-\frac{w_{c}}{W_{c}}\right) d w_{c}\right] \\
& \cdot \frac{1}{W_{u 1}} \exp \left(-\frac{x}{W_{u 1}}\right) d x \\
= & \frac{1}{W_{u 1}} \int_{0}^{\infty} \exp \left[-\left(\frac{R}{W_{c}}+\frac{1}{W_{u 1}}\right) x\right] d x \\
= & \frac{W_{c}}{R W_{u 1}+W_{c}} .
\end{aligned}
$$

Letting $s=W_{u 1} / W_{c},(30)$ becomes

$$
\operatorname{Pr}\left(w_{c}>R w_{u 1}\right)=\frac{1}{R s+1} \text {. }
$$

In Appendix V, it is shown that the pdf of $s$ is

$$
p_{s}(s)=\frac{C}{\sqrt{2} s} \exp \left(-\frac{\log ^{2} s}{2 B}\right) \text {. }
$$

Considering that there are $n$ bits in a packet and averaging over $s$, we get

$$
P_{\text {capt }}(1)=\int_{0}^{\infty} \frac{1}{(R s+1)^{n}} p_{s}(s) d s .
$$

Now the capture probability when the desired packet is overlapped by $I$ interfering packets can be obtained easily:

$$
P_{\text {capt }}(I)=\left\{\int_{0}^{\infty} \frac{1}{(R s+1)^{n}} \frac{C}{\sqrt{2} s} \exp \left(-\frac{\log ^{2} s}{2 B}\right) d s\right\}^{I}
$$

\section{Numerical Results and Discussions}

Fig. 6 shows the system throughput when interleaving is used; that is, each bit in a packet is affected independently by signal fades. There is some improvement in the throughput due to the capture effect, though not significant. This is because there is a chance that the desired packet has higher power than all of other interfering packets over the length of the packet. 


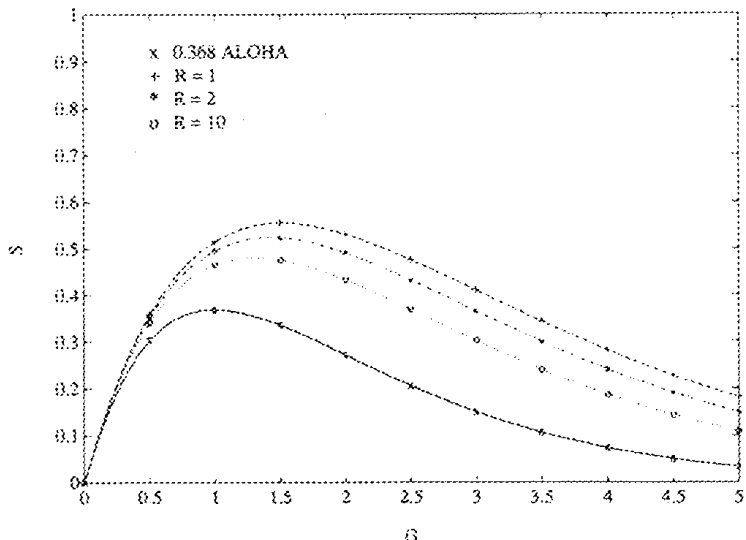

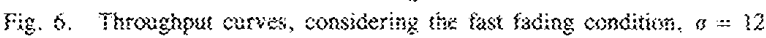

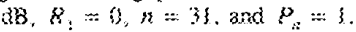

\section{Anplications of Error-Currestion Coding}

Chunel cosing is an effective method for implementing error control in fading chamels, especially under fast fading conditions [19\}. \{20]. To is possible that error-corsection coling may unprove the receiver capture capability, allowing Gurher improvements in the system tixroughpus io be realized. In this subsection, we stwoly the effect of coding on the ALOHA syotem under fast fabing combitions. The result also fulds for slow fading conditions when error worrection coling and interleaving are usaz.

We will hot recommend any speriate yype of code. Our aim is to evaluate the effectiveness of coling in combating the channel fading. Suppose that each nacket consists of a codeword that cun correct un to $t$ errors. The considerai gacket captures the receiver in the presence of $y$ interfering packets with wohability $P$ :am $(l)$ if the namber of erross in the packet is not larges thas: $t$, thus

$$
p_{n: m}(l)=\sum_{j=0}^{b}\left[\begin{array}{l}
n \\
j
\end{array}\right](1 \cdots p)^{j} p^{n \cdots j}
$$

where $p$ is spproximately given by

$$
p \approx p_{i} \prod_{i=1}^{l} \frac{1}{R s_{i}+1},
$$

where $p$ is the probability that a message bil is received comrectly in the presence of tading and shadowing.

In $(36), s_{j}, i=1,2, \cdots, l$, ase mutually independent zandom variables. The pdf of $s$, is the same as $s$ (see (32)). Averaging $\{35\}$ over $s, 0 i \leq 7)$, we have

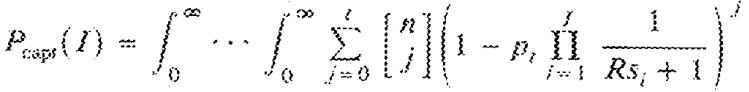

$$
\begin{aligned}
& -\left(p_{i} \prod_{i=1}^{x} \frac{i}{R S_{i}+1}\right)^{n-j} \\
& p_{s_{i}}\left(s_{1}\right) \cdots p_{s j}\left\{s_{s}\right\} d s_{i} \cdots d s_{1}
\end{aligned}
$$

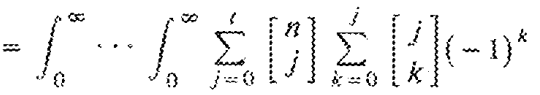

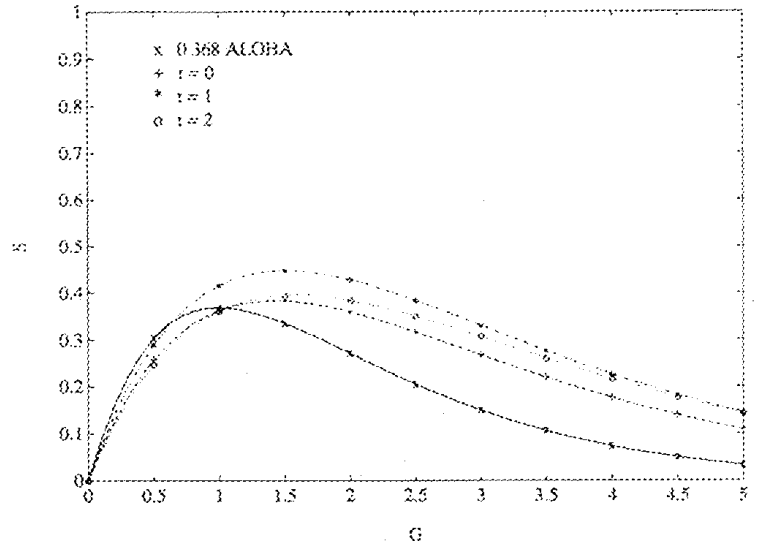

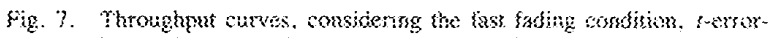

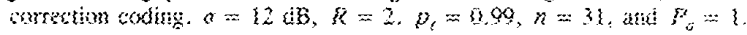

$$
\begin{aligned}
& \cdot\left(\beta_{\gamma} \prod_{i=3}^{i} \frac{1}{K s_{i}+1}\right\}^{k+3 \cdots i} \\
& \cdot p_{s_{2}}\left(s_{3}\right) \cdots p_{n g}\left(s_{l}\right) d s_{1} \cdots d s_{\text {, }} \\
& =\sum_{j=0}^{i}\left\{\begin{array}{l}
n \\
j
\end{array} \sum_{k=0}^{j}\{j](\cdots 1)^{k} p_{t}^{k+\cdots}\right. \\
& \left\{\int_{0}^{\infty} \frac{1}{(k s+1)^{k+n \cdots j} p_{s}(s)}\right. \text {. }
\end{aligned}
$$

Winou conidering the unsuccessuk transmission due 10 acknowledgnent faiuste, we have throughput

$$
\begin{aligned}
& S=c\left\{p ( 0 ) \sum _ { j = 0 } ^ { j } \left\{\begin{array} { l } 
{ n } \\
{ j }
\end{array} \sum _ { k = 0 } ^ { j } \left[\begin{array}{l}
j \\
k
\end{array}(-1)^{k} p_{t}^{k+n j}\right.\right.\right. \\
& +p(1) P_{\text {som }}(1)+p(2) P_{\text {sop }}(2)+\cdots \\
& G=G \exp (-G) \sum_{j=0}^{i}\left[\begin{array}{l}
n \\
j
\end{array} \sum_{k=0}^{j}\left[\begin{array}{l}
j \\
k
\end{array}\right](-1)^{k} p_{i}^{k+n-j}\right. \\
& \cdot \exp \left\{\int_{0}^{\infty} \frac{1}{\left(X_{S}+1\right)^{k+n+i}} \frac{c}{\sqrt{2} s}\right. \\
& \cdot \operatorname{sep}\left(\frac{\log s}{2 B}\right) d s
\end{aligned}
$$

mig. 7 shows that coding enhances the capoure efiect, resulting in increased channel throughnut for ALOHA syssems. $\{31,26,1\}$ and $(31,2\}, 2)$ Bose chandmuri.Hocquenghem (BCH) coles are used in the example. It is worth noting that the implementition of error-onrection tou. ing involves the addition of redundant hits which reduce the effective throughnut of the chamel. When a specific oxe is chosen, the coule rate showd be kaken into consideration in the extimation of aetwork throughpu. la Fig. 7, the ressiting reduction in throwghnut has been considered. Comparng the two curves with $t=3$ and $t=2 \mathrm{in}$ Fig, 7 , it is observed that an increased code power nay not necessurity result in kiofwer througheut because of a becrease in the code rate.

Comparing Figs 6 and 7 with Figs. $\{-3$ and 5 , it is seer: 
that the system under slow fading conditions usually has higher throughput than that under fast fading conditions even though coding is used in the latter case. This can be explained as follows. In slow fading channels where signal power remains constant over the duration of a packet, the probability that a packet has higher power than another packet is 0.5 . On the other hand, in fast fading conditions, the probability that all the bits in a packet have higher power levels than the corresponding bits in another packet is much smaller.

\section{CONCLUSION}

The ALOHA system in the mobile radio environment is investigated in this paper. It is shown that shadowing is similar to fading and near/far phenomena, in that it makes the capture effect possible and provides ALOHA systems with substantial improvements in throughput. It is also confirmed that the superimposed Rayleigh fading, log-normal shadowing, and spatial distribution can further enhance the capture effect, resulting in a ALOHA system with higher throughput. The fast fading effect in ALOHA systems is explored in this paper, we consider a very fast fading case which may be considered to be equivalent to interleaving in slow fading. It is found that the ALOHA system under fast fading conditions also benefits from the capture phenomenon. Improvement in the capture capability results in higher throughput. Further improvements can be made when errorcorrection coding is implemented.

\section{APPENDIX I}

In the Rayleigh fading-only environment, the probability density function of $w_{c}$ is

$$
p_{w_{c}}\left(w_{c}\right)=\frac{1}{W_{0}} \exp \left(-\frac{w_{c}}{W_{0}}\right), \quad w_{c}>0
$$

and $w_{u i}(1 \leq i \leq I)$ has the same distribution with the same mean. The probability in $(2 a)$ can be derived as

$$
\begin{aligned}
\operatorname{Pr}\left(\frac{w_{c}}{\sum_{i=1}^{I} w_{u i}}>R\right) \\
=\int_{0}^{\infty} \cdots \int_{0}^{\infty}\left[\int_{R \sum_{i=1}^{I} x_{i}}^{\infty} \frac{1}{W_{0}} \exp \left(-\frac{w_{c}}{W_{0}}\right) d w_{c}\right] \\
\quad \cdot\left[\prod_{i=1}^{I} \frac{1}{W_{0}} \exp \left(-\frac{x_{i}}{W_{0}}\right)\right] d x_{1} \cdots d x_{I} \\
=\int_{0}^{\infty} \cdots \int_{0}^{\infty} \exp \left(-R \sum_{i=1}^{I} \frac{x_{i}}{W_{0}}\right) \\
\quad \cdot\left[\prod_{i=1}^{I} \frac{1}{W_{0}} \exp \left(-\frac{x_{i}}{W_{0}}\right)\right] d x_{1} \cdots d x_{I} \\
=\int_{0}^{\infty} \cdots \int_{0}^{\infty}\left\{\prod_{i=1}^{I} \frac{1}{W_{0}}\right.
\end{aligned}
$$

$$
\begin{aligned}
& \left.\cdot \exp \left[-\frac{(R+1) x_{i}}{W_{0}}\right]\right\} d x_{1} \cdots d x_{I} \\
& =\frac{1}{(R+1)^{I}} .
\end{aligned}
$$

By assuming $I=1$ and inserting (40) into (4), we find that (2a) agrees with (4).

\section{APPENDIX II}

Assume $x$ and $y$ have the same distribution as shown in (26) and $r=x / y$. The pdf of $r$ is

$$
p_{r}(r)=\int_{0}^{\infty} y p_{x y}(r y, y) d y .
$$

Because $x$ and $y$ are independent, we have

$$
\begin{aligned}
p_{r}(r) & =\int_{0}^{\infty} y p_{x}(r y) p_{y}(y) d y \\
& =\frac{25 x_{0}^{8}}{r^{5}} \int_{0}^{\infty} \frac{1}{y^{9}} \exp \left[-\frac{5}{4}\left(\frac{1}{r^{4}}+1\right) \frac{x_{0}^{4}}{y^{4}}\right] d y
\end{aligned}
$$

After several changes of variables, (42) gives a result in closed form shown in (27).

\section{APPENDIX III}

In (28), considering the integration over $y$ first, we have, after a few steps of derivation,

$$
\begin{aligned}
\int_{0}^{\infty} & \frac{1}{R y^{4} v+u} \frac{y^{3}}{\left(1+y^{4}\right)^{2}} d y \\
& =\frac{1}{4}\left[\frac{1}{u-R v}-\frac{R v}{(u-R v)^{2}} \ln \left(\frac{u}{R v}\right)\right] .
\end{aligned}
$$

Inserting (43) into (28), we get

$$
\begin{aligned}
\operatorname{Pr}\left(w_{c}>R w_{u 1} r^{4}\right)= & C^{2} \int_{0}^{\infty} d u \int_{0}^{\infty} d v \frac{1}{v(u-R v)} \\
& \cdot \exp \left[-\frac{(A-\log u)^{2}}{B}\right] \\
& \cdot \exp \left[-\frac{(A-\log v)^{2}}{B}\right] \\
& -C^{2} \int_{0}^{\infty} d u \int_{0}^{\infty} d v \frac{R}{(u-R v)^{2}} \\
& \cdot \ln \left(\frac{u}{R v}\right) \exp \left[-\frac{(A-\log u)^{2}}{B}\right] \\
& \cdot \exp \left[-\frac{(A-\log v)^{2}}{B}\right] .
\end{aligned}
$$

After changes of variables and using a rotational transformation, similar to the derivation in [7], (29) can be obtained. 
APPENDIX IV

Assume that $W_{c}$ is a local mean with log-normal distribution as shown in (23) and it is attenuated due to the near/far (spatial distribution) phenomenon. We have the probability

$$
\begin{aligned}
& \operatorname{Pr}\left(\frac{W_{c} \beta}{x^{\alpha}}>W\right) \\
& =\operatorname{Pr}\left(W_{c}>\frac{W x^{\alpha}}{\beta}\right) \\
& =\int_{W x^{\alpha} / \beta}^{\infty} \frac{C}{W_{c}} \exp \left[-\frac{\left(\log W_{c}+A\right)^{2}}{B}\right] d W_{c} \\
& =\frac{1}{2} \operatorname{erfc}\left[\frac{\log \left(\frac{W x^{\alpha}}{\beta}\right)+A}{\sqrt{B}}\right],
\end{aligned}
$$

where $x$ is the distance, $\beta$ is a constant, $W$ can be taken as any value not smaller than zero and $\operatorname{erfc}(\cdot)$ is the complementary error function. Equation (45) gives actually the complement of the distribution function of $W_{c}$.

Let $V$ have the same pdf as $W_{c}$ except $V$ takes $A^{\prime}$ as a parameter instead of $A$, where

$$
A^{\prime}=-\log \left(\frac{W_{0} \beta}{x^{\alpha}}\right)+\frac{\sigma^{2}}{2 \log e}
$$

We have

$$
\begin{aligned}
\operatorname{Pr}(V>W) & =\int_{W}^{\infty} \frac{C}{V} \exp \left[-\frac{\left(\log V+A^{\prime}\right)^{2}}{B}\right] d V \\
& =\frac{1}{2} \operatorname{erfc}\left(\frac{\log W+A^{\prime}}{\sqrt{B}}\right) \\
& =\frac{1}{2} \operatorname{erfc}\left[\frac{\log \left(\frac{W x^{\alpha}}{\beta}\right)-\log W_{0}+\frac{\sigma^{2}}{2 \log e}}{\sqrt{B}}\right] \\
& =\frac{1}{2} \operatorname{erfc}\left[\frac{\log \left(\frac{W x^{\alpha}}{\beta}\right)+A}{\sqrt{B}}\right]
\end{aligned}
$$

which gives the same result as (45). This indicates that a log-normal local mean determined by spatial distribution has the same probability property as the local mean (which is not a function of the distance) attenuated due to the near/far phenomenon.

We can further see that a Rayleigh faded signal with mean power varying with distance performs the same as a constant mean Rayleigh signal with attenuation due to the distance, since

$$
\begin{aligned}
\int_{Y}^{\infty} & \frac{x^{\alpha}}{W_{c} \beta} \exp \left(-\frac{w_{c} x^{\alpha}}{W_{c} \beta}\right) d w_{c} \\
= & \int_{Y X^{\alpha} / \beta}^{\infty} \frac{1}{W_{c}} \exp \left(-\frac{v}{W_{c}}\right) d v,
\end{aligned}
$$

where $Y$ is larger than or equal to zero.
Combining the results of (45), (47), and (48), it is concluded that we can study the combined effects of spatial distribution and superimposed Rayleigh and log-normal shadowing by either considering that the log-normal local mean varies with the distance or assuming that the local mean does not depend on the distance and using the distance as an attenuation factor to the received signal.

$$
\begin{gathered}
\text { APPEndix V } \\
p_{s}(s)=\int_{0}^{\infty} W_{c} p_{W_{u 1} W_{c}}\left(s W_{c}, W_{c}\right) d W_{c} \\
=\frac{C^{2}}{s} \int_{0}^{\infty} \frac{1}{W_{c}} \exp \left[-\frac{\left(\log s W_{c}+A\right)^{2}}{B}\right] \\
\cdot \exp \left[-\frac{\left(\log W_{c}+A\right)^{2}}{B}\right] d W_{c} .
\end{gathered}
$$

Let $W_{\mathrm{c}}^{\prime}=\log W_{c},(49)$ becomes

$$
\begin{array}{r}
p_{s}(s)=\frac{C^{2}}{s \log e} \int_{-\infty}^{\infty} \exp \left\{-\frac{2}{B}\left[\left(W_{c}^{\prime}+A+0.5 \log s\right)^{2}\right.\right. \\
\left.\left.+0.25 \log ^{2} s\right]\right\} d W_{c}^{\prime}, \quad(50)
\end{array}
$$

which gives the result as shown in (32).

\section{ACKNOWLEDGMENT}

The authors express their thanks to the reviewers of this paper for their valuable comments.

\section{REFERENCES}

[1] N. Abramson, "The ALOHA system-Another alternative for computer communications," in Proc. 1970 Fall Joint Comput. Conf., 1970 , pp. $281-285$

[2] - "The throughput of packet broadcasting channels," IEEE Trans. Commun., vol. COM-25, pp. 117-128, Jan. 1977.

[3] J. A. Roberts and T. J. Healy, "Packet radio performance over slow Raleigh fading channels," IEEE Trans. Commun., vol. COM-28, pp. 279-286, Feb. 1980 .

[4] J. J. Metzner, "On improving utilization in ALOHA networks,' IEEE Trans. Commun., vol. COM-24, pp. 447-448, Apr. 1976.

[5] F. Kuperus and $J$. Arnbak, "Packet radio in a Rayleigh channel," Electron. Lett., vol. 18, no. 12, pp. 506-507, June 1982

[6] J. Ambak and W. Blitterswijk, "Capacity of slotted ALOHA in Rayleigh-fading channels," IEEE J. Select. Areas Commun., vol. SAC-5, pp. 261-269, Feb. 1987.

[7] E. Zainal and R. Garcia, "The effects of Rayleigh fading on capture phenomenon in ALOHA channels," in Proc. INFOCOM'87, Mar. 1987 , pp. 888-893.

[8] C. Namislo, "Analysis of mobile radio slotted ALOHA networks," IEEE Trans. Veh. Technol., vol. VT-33, pp. 199-204, Aug. 1984.

[9] D. J. Goodman and A. A. M. Saleh, "The near/far effect in local ALOHA radio communications," IEEE Trans. Veh. Technol., vol. VT-36, pp. 19-27, Feb. 1987

[10] R. Prasad and J. Arnbak, "Enhanced throughput in packet radio channels with shadowing," Electron. Lett., vol. 24, no. 16, pp. 986-988, Aug. 1988.

[11] _ , "Enhanced throughput in packet radio channels with fading and shadowing," in Proc. Canadian Conf. Elec. Comput. Eng., pp. 78-80, Nov. 1988.

[12] W. C. Y. Lee, Mobile Communications Engineering. New York: McGraw-Hill, 1982

[13] L. G. Roberts, "ALOHA packet system with and without slots and capture," Comput. Commun. Rev., vol. 5, pp. 28-42, Apr. 1975. 
[14] L. Fratta and D. Sant, "Some models of packet radio networks with capture," in Proc. Fifth Int. Conf. Comput. Commun., 1980, pp. 155-161.

[15] R. Nelson and L. Kleinrock, "The spatial capacity of a slotted ALOHA multihop packet radio network with capture," IEEE Trans. Commun., vol. COM-32, pp. 684-694, June 1984.

[16] F. Hansen and F. I. Meno, "Mobile fading-Rayleigh and Log-normal superimposed," IEEE Trans. Veh. Technol., vol. VT-26, pp. 332-335, Nov. 1977.

[17] R. C. French, "The effect of fading and shadowing on channel resue in mobile radio," IEEE Trans. Veh. Technol., vol. VT-28, pp. 171-181, Aug. 1979.

[18] S. A. Musa and W. Wasylkiwskyj, "Co-channel interference of spread spectrum systems in a multiple user environment," IEEE Trans. Commun., vol. COM-26, pp. 1405-1413, Oct. 1978.

[19] W. C. Y. Lee, "The advantages of using repetition code in mobile radio communications," in Proc. IEEE Veh. Technol. Conf., May 1986, pp. 157-161.

[20] Y. D. Yao and A. U. H. Sheikh, "Diversity and coding techniques for digital mobile radio communications," in Proc. Third Nordic Seminar on Digital Land Mobile Radio Commun., Sept. 1988.

[21] I. S. Gradshteyn and I. M. Ryzhik, Table of Integrals, Series, and Products. New York: Academic, 1980.

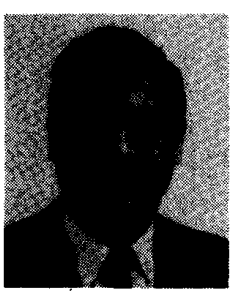

Asrar U. H. Sheikh (M'71-M'83-SM'86) graduated from the University of Engineering and Technology, Lahore, Pakistan, and received the M.Sc. and Ph.D. degrees from the University of Birming ham, England, in 1966 and 1969, respectively.

He held Lectureships at universities in Pakistan, Iran, and Libya between 1969 and 1975 . In 1975 he returned to the University of Birmingham as a Postdoctoral Fellow to work on the characterization of man-made noise. In 1978, he returned to Libya as an Associate Professor at Garyomis University. He joined Carleton University, Ottawa, ON, Canada, as an Associate Professor in 1981, and is currently a Professor and Associate Chairman responsible for graduate studies. His current interests in communication systems cover digital cellular systems, packet radio, spread spectrum, mobile and portable networks and signal processing.
Dr. Sheikh was co-recipient of the IERE Paul Adorian Premium awarded for best papers published in the area of broadcasting engineering in 1983 . He has provided technical consulting services to a number of public and private organizations. He is member of the Association of Professional Engineers of Ontario.

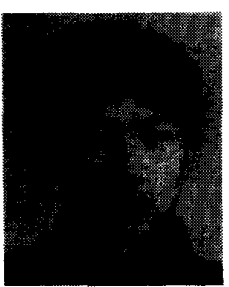

Yu-Dong Yao (S'88-M'88) was born in Tianjin, China, on November 22, 1960 . He received the B.Eng. and M.Eng. degrees from Nanjing University of Posts and Telecommunications, Nanjing, China, in 1982 and 1985 , respectively, and the Ph.D. degree from Southeast University, Nanjing, China, in 1988, all in electrical engineering.

From 1987 to 1988 , he was at Carleton University, Ottawa, ON, Canada, completing his Ph.D. program. Since 1989 , he has been with Carleton University as a Research Associate working on mobile radio communications and digital signal processing for ISDN access. He holds a patent on telephony. His current research interests include mobile radio, spread spectrum, error-correction coding, digital signal processing and communication protocols.

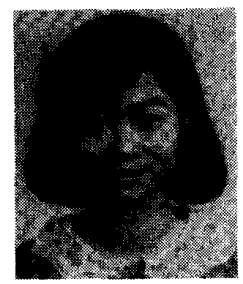

Xiaoping Wu (S'90) was born in Nanjing, China. She received the B.Eng. degree in electrical engineering from Beijing University of Posts and Telecommunications, Beijing, China, in 1985.

From 1985 to 1988 she was with Nanjing University of Posts and Telecommunications, Nanjing, as a teacher and researcher. Since 1988, she has been studying for the M.Eng. degree in electrical engineering, first at Concordia University, Montreal, PQ, Canada, and currently at Carleton University, Ottawa, ON, Canada. Her research interests include computer communications, packet radio networks and broad-band ISDN.

Ms. Wu is a student member of the IEEE Communications, Information Theory, and Vehicular Technology Societies. 